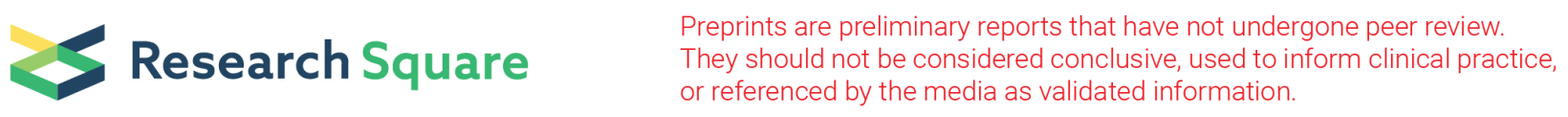

\title{
Door-to-door recruitment during the COVID-19 pandemic: Lessons learned from a population- based, longitudinal cohort study in North Carolina, USA
}

Jaclyn Karasik ( $\sim$ jkarasik@unc.edu )

Research in practice

Keywords: epidemiology, prospective cohort, recruitment methods, SARS-CoV-2, seroprevalence

Posted Date: February 17th, 2022

DOI: https://doi.org/10.21203/rs.3.rs-1234834/v1

License: (c) (1) This work is licensed under a Creative Commons Attribution 4.0 International License. Read Full License 


\section{Abstract \\ Background}

The COVID-19 pandemic has disproportionately impacted many of the same communities that have been historically harmed by or underrepresented in public health research. In a prospective cohort study on COVID-19 in North Carolina, USA, we employed a door-to-door recruitment strategy on a randomly selected sample of households to maximize inclusivity and improve study diversity.

\section{Discussion}

Rapidly shifting current events and an evolving pandemic required continuous updating of our approach. Using a variety of recruitment strategies and participation methods allowed us to quickly adapt and to reach a broad range of people with diverse needs and circumstances. Door-to-door recruitment had many unexpected benefits, allowing us to easily reach people that were working from home and leaving people with positive impressions of public health research. However, even when we were able to elicit a response from prospective participants, follow-up has remained a substantial challenge.

\section{Conclusions}

It is paramount for public health practitioners to employ creative strategies and to invest time and resources to include hard-to-reach communities in research.

\section{Background}

In the United States, the COVID-19 pandemic has had a disproportionate impact on socio-economically disadvantaged and rural communities, which suffer from longstanding health disparities and limited access to care (1). Therefore, it is critical to ensure that these individuals are represented in epidemiological studies and clinical trials, despite the fact that these communities have been historically under-represented in public health research $(2,3)$.

We conducted a population-based, longitudinal cohort study to estimate incidence and community prevalence of SARS-CoV-2 infections in Chatham County, North Carolina (4). Initial recruitment efforts included 1536 addresses selected in a stratified, two-stage probability-based sampling design. All sampled households received postcards or emails; those with phone numbers who did not respond to mail were given phone calls. Door-to-door home visits were attempted among a subset of those who still did not respond to postcards or phone calls.

Despite oversampling in regions of the county with lower median income levels and/or higher concentrations of Hispanic/Latino and Black/African American populations, interim analysis of our participants after five months of postcard and phone recruitment suggested that our cohort did not reflect 
the demographics of Chatham County (4). In brief, the cohort was skewing toward older, wealthier, and predominantly White participants. We hypothesized that our recruitment efforts, initially limited by concerns regarding shortages of personal protective equipment and restrictions on face-to-face interactions, would not yield a representative cohort due to differential nonresponse across population subgroups $(5,6)$. As those concerns subsided, we implemented a door-to-door recruitment approach in February 2021 to increase representation within the cohort to better reflect the underlying population. In this paper, we share challenges and lessons we have learned along the way while recruiting for this study.

\section{Lesson 1: Current Events Affected Recruitment}

Monitoring current events was key to anticipating and successfully navigating barriers to study recruitment. Initial recruitment efforts benefited from contacting sampled individuals at a time when there was a widespread feeling of powerlessness. Individuals frequently perceived participation in the study as an opportunity to "help fight COVID-19." However, we observed a clear decrease in response to mailings and phone calls during the lead up to the 2020 United States presidential election. This was a time when many residents were already receiving high volumes of election-related mail and phone calls. After this subsided, enthusiasm continued to wane with the rapid roll-out of vaccines and the onset of "pandemic fatigue" (7).

Some challenges remained consistent throughout the recruitment period. Political ideology affected how people adapted COVID-19 preventative behaviors and mistrust in public health authorities remained widespread $(8,9)$. Recruitment attempts by study staff were frequently met with skepticism and even dismissiveness from individuals choosing not to adhere to preventative measures such as masking or social distancing.

In addition, it was difficult to engage local organizations in recruitment efforts as many were already stretched thin while responding to a surge in community need, including food and economic insecurity. Asking staff to assist with door-to-door recruitment would have placed additional burden on these organizations already responding to a crisis. Community organizations were able to provide some consultation in the development of the study, and we were able to provide gift cards to support their work.

\section{Lesson 2: Recruitment Methods Changed With Greater Understanding Of Transmission Modes}

Knowledge of SARS-CoV-2 transmission modes and dynamics were still uncertain when study recruitment efforts began in July 2020. Community engagement is a critical aspect of public health research (10), but in-person interactions at local community centers and churches were initially limited 
due to lockdowns and concerns for study staff safety. Once the team began door-to-door recruitment, concerns regarding spread via surfaces (e.g., tablets, pencils, etc.) and close contact during conversations necessitated meticulously designed and rehearsed door-to-door recruitment protocols. For example, we began by only collecting contact information of interested participants in order to limit face-to-face contact time while visiting homes, even as most encounters were conducted outside and at distance of at least six feet. Unfortunately, the limited information often led to attrition when staff attempted to follow up at a later time to complete the consent process by phone. Once study staff were vaccinated and more evidence regarding the limited potential of outdoor transmission emerged, we found that being able to complete the full consent process at their homes helped to secure enrollment. In short, our recruitment methods needed to adapt to the changing state-of-knowledge.

\section{Lesson 3: Diverse Methods Yielded Diverse Participants}

Chatham County encompasses both rural and semi-urban towns, a large Spanish-speaking and African American population, and populations of highly variable socioeconomic status and age ranges, including working families to retirees. The county also spans a large geographic area (18th largest out of 100 counties in North Carolina). Employing diverse recruitment methods meant that we reached out to people through email, postal mail, phone calls, and door-to-door visits to homes. This was especially important during the COVID-19 pandemic when the digital divide caused additional barriers to resources for many (11). We also offered diverse ways to participate in the study to maximize inclusivity and offset concerns about transmission risk in public spaces. We offered to collect blood and nasal swab samples at an established health clinic in the vicinity of their home (up to 20 minutes away by car) or allowed participants to self-collect samples using at-home sampling devices for both blood and nasal swabs. Similarly, we offered online and written mail-in surveys for those without internet or email. Thus, those who were not comfortable going out in public due to the threat of COVID-19 or lack of transportation were still able to participate without leaving their homes.

In addition, diversity in our field staff (African American, White, and Spanish-speaking/Latinx) reflected demographics of Chatham County and helped to build trust with potential participants. We also found that introducing ourselves as students was a disarming icebreaker that opened up the conversation enough for us to explain the purpose for our visit. We found that Spanish-speaking potential participants often became much more interested in learning about our work once they realized that a field staff member spoke Spanish. Research staff also worked to raise awareness about the study more broadly in the community by handing out bilingual flyers at a holiday market and working with community partners. In brief, employing a range of recruitment methods, offering multiple avenues to participate in the study, and having a diverse team of field staff helped to support recruitment of diverse participants that reflected the demography of Chatham.

\section{Lesson 4: Follow-up Remained A Challenge}


In addition to challenges with enrollment, it was also difficult to keep participants retained and engaged at all phases of the study. Several individuals consented to participate but never made it to their first clinic visit. While our monthly clinic visits only lasted about 15 minutes, our clinics are held on weekdays from 8 am-5pm, which posed challenges for those with less freedom to take time off work. We offered mail-in test kits to attempt to address this issue, but many participants still had difficulties making time for the study.

An unanticipated challenge in recruiting participants involved cultural considerations. Several households offered their contact information out of what we now perceive as courtesy or politeness but then were difficult to reach to complete the enrollment process. As a result, we switched to enrolling participants while visiting their homes to reduce attrition. We also emphasized that the study was voluntary, and they were not obligated to give their contact information if uninterested. Enacting this new strategy resulted in consenting 11 participants on the spot.

Door-to-door successfully yielded participants that were younger, had a lower income, and were more diverse in terms of race and ethnicity in comparison to participants recruited through other methods (Table 1; Figure 1). This was expected because we targeted our door-to-door recruitment geographically to improve reach of persons with these demographics. It also resulted in similar enrollment yield compared with other methods (Figure 2), with 4.6\% ( $n=23$ ) of homes visited enrolling in the study. However, door-todoor efforts did not yield enough participants to significantly increase the size or overall diversity of our cohort by race, ethnicity, or age. We anticipated this difficulty with enrolling homes in the door-to-door recruitment stage since they had already declined to respond to mailings and phone calls.

Unfortunately, we also have seen the greatest rate of withdrawal among participants recruited with the door-to-door method compared to others. Many of these participants missed initial visits and study staff put in extra effort to confirm appointments with participants to ensure the best chance of adherence. As of November 2021 , about $43 \%$ of door-to-door participants have withdrawn compared to $16 \%$ of participants recruited by phone and $17 \%$ of postcard/email participants. We expected this as many of the door-to-door participants are socio-economically disadvantaged and likely to face additional barriers to participation and retention in research, compared to participants from socio-economically advantaged groups (12). Some barriers identified in previous reviews include mistrust in research and the medical system, limited transportation, time and financial constraints, and family obligations $(13,14)$.

\section{Table 1. Demographics of Chatham County, NC COVID-19 Cohort participants $(n=153)$, stratified by method of recruitment}




\begin{tabular}{|c|c|c|c|c|}
\hline & $\begin{array}{l}\text { Postcard or } \\
\text { Email ( } n=81 \text { enrolled } \\
\text { participants) }\end{array}$ & $\begin{array}{l}\text { Phone Call } \\
(n=49)\end{array}$ & $\begin{array}{l}\text { Door to } \\
\text { Door } \\
(n=23)\end{array}$ & $\begin{array}{l}\text { All } \\
\text { participants } \\
(n=153)\end{array}$ \\
\hline $\begin{array}{l}\text { White Non-Hispanic } \\
n \text { / total responses (\%) }\end{array}$ & $62 / 77(80.5 \%)$ & $\begin{array}{l}38 / 43 \\
(88.3 \%)\end{array}$ & $\begin{array}{l}6 / 15 \\
(40 \%)\end{array}$ & $\begin{array}{l}106 / 135 \\
(78.5 \%)\end{array}$ \\
\hline $\begin{array}{l}\text { Median Years of Age } \\
(Q 1, Q 3)\end{array}$ & $63(51,70)$ & $60(47,67)$ & $\begin{array}{l}45(35, \\
59)\end{array}$ & $61(47,68)$ \\
\hline $\begin{array}{l}\text { Income } n(\%) \\
\text { less than } \\
\$ 49,999 \\
\$ 50,000-\$ 74,999 \\
\$ 75,000 \text { or more } \\
\text { Missing }\end{array}$ & $\begin{array}{l}(17.3 \%) \\
(25.9 \%) \\
(50.6 \%) \\
(6.2 \%)\end{array}$ & $\begin{array}{ll}9 & (18.4 \%) \\
9 & (18.4 \%) \\
27 & (55.1 \%) \\
4 & (8.2 \%)\end{array}$ & $\begin{array}{ll}9 & (39.1 \%) \\
3 & (13.0 \%) \\
3 & (13.0 \%) \\
8 & (34.8 \%)\end{array}$ & $\begin{array}{l}(20.9 \%) \\
(21.6 \%) \\
(46.4 \%) \\
(11.1 \%)\end{array}$ \\
\hline
\end{tabular}

Table 1. All demographic information was collected prior to the participant's first visit or sample collection via email or paper surveys for participants without email.

\section{Lesson 5: In-person Recruitment Had Unexpected Benefits}

With many people spending more time at home during the pandemic, it was often more accessible to reach participants at home on the weekdays in addition to weekends. Even when households were not interested in participating, they frequently expressed a sense of appreciation for our study and for the time spent traveling to their home. For example, one household shared their reservations about the COVID-19 vaccines and the source of the pandemic, but still positively commented on our efforts to serve Chatham County and asked thoughtful questions about our work. Thus, even when households did not participate, our encounters provided Chatham County residents with the opportunity to develop a positive perception and awareness of research efforts. Ideally, this could increase willingness to participate in future studies.

\section{Conclusion}

Conducting a longitudinal, population-based cohort study of a highly transmissible respiratory virus in the midst of global pandemic presented many challenges, especially when attempting to recruit and retain a representative cohort including diverse populations. Our first-hand experience highlighted how secular events may influence possible participants towards or against research. Similarly, our enrollment patterns demonstrate how recruitment strategies must be responsive to the needs of the target population. No single method can achieve adequate representation in population-based studies, and multi-mode data collection allows for recruitment of a more diverse population. In conclusion, lessons learned from our ongoing study will inform future community-based research during quickly evolving public health threats.

\section{Abbreviations}

coronavirus disease 2019 (COVID-19), severe acute respiratory syndrome coronavirus 2 (SARS-CoV-2) 


\section{Declarations}

- Ethics approval and consent to participate: Ethical approval was provided by the University of North Carolina at Chapel Hill Institutional Review Board (IGHID 12029, IRB\#20-1632). All protocols were carried out in accordance with relevant guidelines and regulations. Informed consent was obtained from all participants.

- Consent for publication: Not applicable

- Availability of data and materials: The datasets used and analyzed during the current study are available from the corresponding author on reasonable request.

- Competing Interests: The authors declare that they have no competing interests.

- Funding: All authors report funding for seroprevalence studies from the North Carolina Department of Health and Human Services. RMB is supported by the National Institutes of Health (K23Al141764). BES receives support from the University of North Carolina at Chapel Hill Center for AIDS Research (CFAR), a National Institutes of Health funded program P30 Al050410. AEA received research support from NICHD and NIA of the National Institutes of Health under award numbers P2C HD050924 and P30 AG066615, respectively. The content is solely the responsibility of the authors and does not necessarily represent the official views of the National Institutes of Health. This work was supported by a contract between the University of North Carolina at Chapel Hill and the North Carolina Department of Health and Human Services, Division of Public Health (https://publichealth.nc.gov/), contract 00041877 awarded to author RB. The funders did not have and will not have a role in study design, data collection and analysis, decision to publish, or preparation of the manuscript.

- Authors' contributions: J.M.K., C.M., and E.A.L. wrote the initial draft of the paper. J.M.K., C.M., E.A.L., and K.X. implemented the recruitment methods. M.B. led community partner engagement efforts. J.M.K. and C.M. analyzed data and prepared figures. A.E.A., B.E.S., and R.M.B. conceived of and led the study. All authors contributed to editing and revising the paper and have approved the final manuscript.

Acknowledgements: The authors thank the participants for their willingness to contribute to advancing our understanding of the SARS-CoV-2 epidemic and its impact on rural communities, especially during the early and uncertain months of the pandemic.

\section{References}

1. Karmakar M, Lantz PM, Tipirneni R. Association of Social and Demographic Factors With COVID-19 Incidence and Death Rates in the US. JAMA Netw Open. 2021 Jan 4;4(1):e2036462.

2. Freedman LS, Simon R, Foulkes MA, Friedman L, Geller NL, Gordon DJ, et al. Inclusion of women and minorities in clinical trials and the NIH Revitalization Act of 1993-the perspective of NIH clinical 
trialists. Control Clin Trials. 1995 Oct;16(5):277-85. discussion 286.

3. Corbie-Smith GM. Minority recruitment and participation in health research. N C Med J. 2004 Dec;65(6):385-7.

4. Miller EM, Law EA, Ajeen R, Karasik J, Mendoza C, Abernathy $H$, et al. SARS-CoV-2 infection in central North Carolina: Protocol for a population-based longitudinal cohort study and preliminary participant results. PLoS ONE. 2021 Oct;25(10):e0259070. 16(.

5. Ejiogu N, Norbeck JH, Mason MA, Cromwell BC, Zonderman AB, Evans MK. Recruitment and retention strategies for minority or poor clinical research participants: lessons from the Healthy Aging in Neighborhoods of Diversity across the Life Span study. Gerontologist. 2011 Jun;51(Suppl 1):33-45.

6. Galea S, Tracy M. Participation rates in epidemiologic studies. Ann Epidemiol. 2007 Sep;17(9):64353.

7. Haktanir A, Can N, Seki T, Kurnaz MF, Dilmaç B. Do we experience pandemic fatigue? current state, predictors, and prevention. Curr Psychol. 2021 Oct;20:1-12.

8. Harvard TH. Chan School of Public Health. The Public's Perspective on the United States Public Health System. May: Robert Wood Johnson Foundation; 2021.

9. Han Q, Zheng B, Cristea M, Agostini M, Bélanger JJ, Gützkow B, et al. Trust in government regarding COVID-19 and its associations with preventive health behaviour and prosocial behaviour during the pandemic: a cross-sectional and longitudinal study. Psychol Med. 2021 Mar;26:1-11.

10. Sapienza JN, Corbie-Smith G, Keim S, Fleischman AR. Community engagement in epidemiological research. Ambul Pediatr. 2007 Jun;7(3):247-52.

11. Clare CA. Telehealth and the digital divide as a social determinant of health during the COVID-19 pandemic. Netw Model Anal Health Inform Bioinform. 2021 Apr 3;10(1):26.

12. Bonevski B, Randell M, Paul C, Chapman K, Twyman L, Bryant J, et al. Reaching the hard-to-reach: a systematic review of strategies for improving health and medical research with socially disadvantaged groups. BMC Med Res Methodol. 2014 Mar;25:14:42.

13. George S, Duran N, Norris K. A systematic review of barriers and facilitators to minority research participation among African Americans, Latinos, Asian Americans, and Pacific Islanders. Am J Public Health. 2014 Feb;104(2):e16-31.

14. Ford JG, Howerton MW, Lai GY, Gary TL, Bolen S, Gibbons MC, et al. Barriers to recruiting underrepresented populations to cancer clinical trials: a systematic review. Cancer. 2008 Jan 15;112(2):228-42.

\section{Figures}


POSTCARD OR EMAIL

PHONE CALL

DOOR TO DOOR

TOTAL COHORT
$17.3 \%$

$25.9 \%$

$50.6 \%$

$6.2 \%$

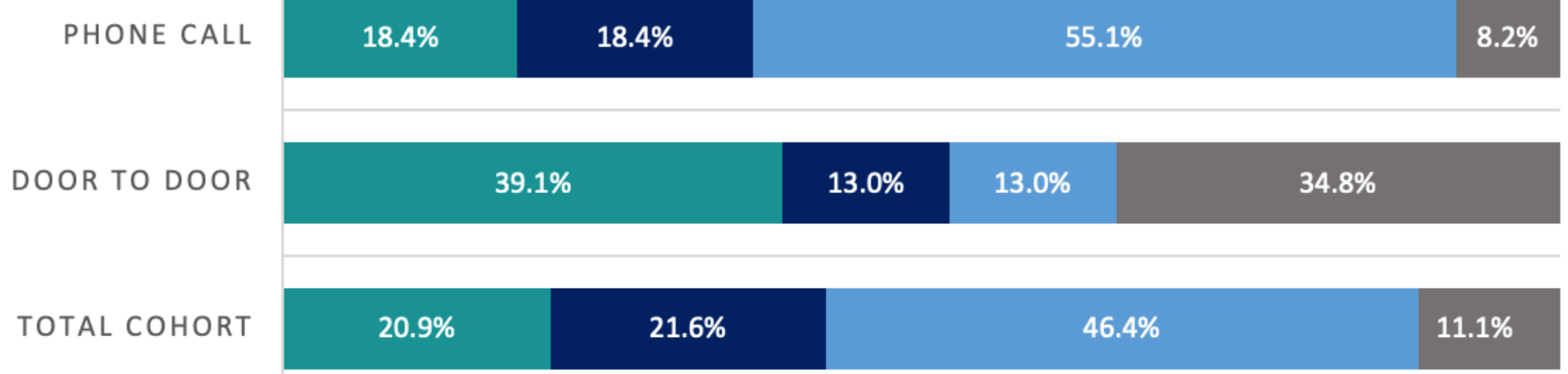

Figure 1

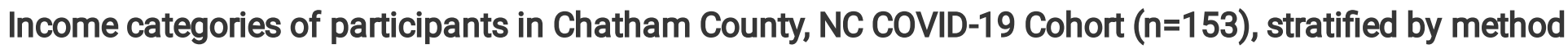
of recruitment

- Enrolled

- Opted out or expressed interest but never enrolled

- Unreachable or no response

POSTCARD OR EMAIL

$(\mathrm{N}=1536)$

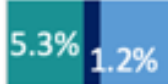

$93.6 \%$

PHONE CALL

$(\mathrm{N}=1002)$

DOOR-TO-DOOR

$(N=500)$

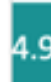

$27.4 \%$

$67.7 \%$

$60.8 \%$

\begin{tabular}{|c|c|c|c|c|}
\hline & Enrolled & $\begin{array}{l}\text { Opted out or expressed } \\
\text { interest; never enrolled }\end{array}$ & $\begin{array}{l}\text { Unreachable or } \\
\text { no response }\end{array}$ & Total \\
\hline Postcard or email n (\%) & $81(5.3 \%)$ & $18(1.2 \%)$ & $1416(60.8 \%)$ & 1536 \\
\hline Phone call n (\%) & $49(4.9 \%)$ & $275(27.4 \%)$ & $678(67.7 \%)$ & 1002 \\
\hline Door-to-door n (\%) & $23(4.6 \%)$ & $116(23.2 \%)$ & $304(60.8 \%)$ & 500 \\
\hline
\end{tabular}

Figure 2

Chatham County COVID-19 Cohort Study Recruitment Outcomes (sample size of n=1536) All eligible households in the sample were contacted via postcard or email. Households with available phone numbers were then given phone calls. Households that had still failed to respond were contacted via door-to-door outreach; not all non-responsive households were contacted due to resource limitations. 